\title{
Notch1 haploinsufficiency causes ascending aortic aneurysms in mice
}

\author{
Sara N. Koenig, ${ }^{1,2,3}$ Stephanie LaHaye, ${ }^{1,2,4}$ James D. Feller, ${ }^{1,2}$ Patrick Rowland, ${ }^{1,2}$ Kan N. Hor, ${ }^{2,5}$ \\ Aaron J. Trask,, ${ }^{1,2,5}$ Paul M.L. Janssen, ${ }^{6}$ Freddy Radtke, ${ }^{7}$ Brenda Lilly, ${ }^{1,2,4}$ and Vidu Garg ${ }^{1,2,3,4,5}$ \\ 'Center for Cardiovascular Research and ${ }^{2}$ The Heart Center, Nationwide Children's Hospital, Columbus, Ohio, USA. \\ ${ }^{3}$ Dorothy M. Davis Heart and Lung Research Institute, ${ }^{4}$ Department of Molecular Cenetics, ${ }^{5}$ Department of Pediatrics, \\ and ${ }^{6}$ Department of Physiology and Cell Biology, The Ohio State University, Columbus, Ohio, USA. ' $E c$ cole Polytechnique \\ Fédérale de Lausanne, Swiss Institute for Experimental Cancer Research, Lausanne, Switzerland.
}

\begin{abstract}
An ascending aortic aneurysm (AscAA) is a life-threatening disease whose molecular basis is poorly understood. Mutations in NOTCH1 have been linked to bicuspid aortic valve (BAV), which is associated with AscAA. Here, we describe a potentially novel role for Notch1 in AscAA. We found that Notch1 haploinsufficiency exacerbated the aneurysmal aortic root dilation seen in the Marfan syndrome mouse model and that heterozygous deletion of Notch1 in the second heart field (SHF) lineage recapitulated this exacerbated phenotype. Additionally, $\mathrm{Notch}^{+/-}$mice in a predominantly 12956 background develop aortic root dilation, indicating that loss of Notch1 is sufficient to cause AscAA. RNA sequencing analysis of the Notch1.12956 ${ }^{+/-}$aortic root demonstrated gene expression changes consistent with AscAA. These findings are the first to our knowledge to demonstrate an SHF lineage-specific role for Notch1 in AscAA and suggest that genes linked to the development of BAV may also contribute to the associated aortopathy.
\end{abstract}

Conflict of interest: The authors have declared that no conflict of interest exists.

Submitted: November 3, 2016 Accepted: September 29, 2017 Published: November 2, 2017

Reference information: JCI Insight. 2017;2(21):e91353. https:// doi.org/10.1172/jii.insight.91353.

\section{Introduction}

Thoracic aortic aneurysms (TAA) have an incidence of approximately 9-16 per 100,000 people per year (1) and are associated with a significant morbidity and mortality due to the asymptomatic nature of the disease and minimal available therapeutic interventions. Aneurysmal dilation of the aorta is accompanied by pathologic changes including loss of smooth muscle cells (SMCs), dysregulation of the extracellular matrix (ECM), and degradation of the elastic laminae (2). These changes prohibit the vessel from maintaining normal compliance and ultimately result in dissection or rupture of the aorta. TAAs are typically found in associated syndromic populations including Marfan syndrome (MFS), caused by mutations in FBN1 (3-6); Loeys-Deitz syndrome, caused by mutations in TGFBR1, TGFBR2, TGFB2, TGFB3, and SMAD3 (7-10); Fabry disease, caused by mutations in GLA (11); Turner syndrome, associated with monsomy of chromosome X (12); and Ehlers-Danlos syndrome, caused by mutations in Col3A1 (13). Additional genetic links to TAA have also been reported in families harboring diseasesegregating mutations in ACTA2 (14), MYH11 (15), LOX (16, 17), and FOXE3 (18).

Approximately half of TAAs occur in the portion of the aorta that precedes the brachiocephalic trunk, the ascending aorta (Figure 1A). Ascending aortic aneurysms (AscAA) are commonly associated with bicuspid aortic valve (BAV), a developmental defect that arises from incomplete separation or fusion of 2 of 3 aortic valve cusps. The etiology of AscAA in the setting of BAV has been debated (19). BAV or thickening of valve cusps resulting in stenosis can alter hemodynamics in the ascending aorta, either by changing patterns of blood flow, which alters shear stress, or by increasing velocity of blood flow, which increases normal stress, leading to aortopathy characterized by aortic dilation (reviewed in ref. 20). However, the progressive dilation of the aorta and development of AscAA that has been reported even after aortic valve replacement cannot be accounted for by hemodynamic changes alone, suggesting other potential mechanisms (21). Interestingly, 2 progenitor cell populations, the second heart field (SHF) and the cardiac neural crest (CNC), contribute to the development of the aortic valve and the ascending aorta (22-26) (Figure 1A). Therefore, we hypothesized genetic mutations that cause BAV may also contribute to abnormal development of the ascending aorta and predispose to the development of aortopathy in individuals with BAV. 
Mutations in NOTCH1 were first identified in patients with a BAV and associated aortic valve calcification, but even among these initial families, a subset had associated aortopathy (27). Subsequently, there have been multiple reports of NOTCH1 mutations in patients with BAV and associated aortopathy (28-30). NOTCH1 encodes one of the 4 transmembrane receptors (NOTCH1-4) that functions in the highly conserved Notch signaling pathway, which is a critical player in cardiovascular development and disease. A signaling cell presents a NOTCH ligand from the Jagged and Delta-like families (JAGGED1-2, DELTA-LIKE1,-3, and -4) for binding to the extracellular domain of a NOTCH receptor. After ligand binding, the NOTCH intracellular domain (NICD) is activated after a series of proteolytic cleavages, ultimately initiating transcription of downstream target genes. Notch1 is critical for development, as homozygous-null mice die at embryonic day 10.5 (E10.5) due to vascular defects $(31,32)$. Notch1 is expressed in the developing cardiac outflow tract and has been shown to be required for proper semilunar valve development (27, 33 , 34). Furthermore, loss of Notch signaling in the SHF or CNC, the 2 progenitor cell populations that form the cardiac outflow tract, results in abnormal development of the aorta and pulmonary artery $(33,35)$. We recently reported a highly penetrant mouse model for BAV by introducing Notch1 heterozygosity into an endothelial nitric oxide synthase-null (Nos3-null) background (34). These compound mutant mice develop features of aortic dilation with early effacement of the sinotubular junction (STJ), defined as a loss of a distinct border between the aortic sinus and proximal ascending aorta (36). Pathologic features consistent with aortic aneurysm development were also noted in the absence of significant aortic valve stenosis, suggesting that Notch1 signaling may contribute to AscAA. Additional work by us and others demonstrated that loss of Notch1 in the endothelial cell lineage contributes to BAV and abnormal valve remodeling $(37,38)$.

To determine if Notch1 has a role in the development of AscAA that is independent of BAV, we utilized a well-described murine model of AscAA, the MFS mouse (Fbn1 ${ }^{\mathrm{C1039G} /+}$ ) (39). This model allowed us to test the hypothesis that loss of genes involved in aortic valve development, such as Notch1, contribute to the pathogenesis of AscAA. Here, we show that heterozygous deletion of Notch1 exacerbates the AscAA phenotype observed in the MFS mouse. In addition, we found that haploinsufficiency of Notch1 in the SHF lineage — but not the mature smooth muscle, endothelial, or CNC lineages — is sufficient to cause this exacerbated phenotype. Furthermore, Notch $1^{+/-}$mice that were backcrossed 5 generations in a 129S6 background develop aortic root dilation in the absence of BAV. While RNA sequencing (RNA-seq) analysis demonstrated differential gene expression between the aortic root and distal ascending aorta, it also demonstrated gene expression changes in the aortic root of Notch1.129S6 $6^{+/-}$mice, consistent with aortopathy. Overall, our findings are the first to our knowledge to demonstrate an SHF-specific role for Notch1 in AscAA.

\section{Results}

Heterozygosity of Notch1 exacerbates the AscAA in MFS mice. Based on previously published evidence suggesting the potential role for Notch1 in AscAA $(27-30,36,40)$, we sought to determine if loss of Notch1 would increase the rate of aortic dilation in a mouse model of AscAA. The MFS mouse harbors a human MFS-causing mutation in Fibrillin1 (FBN1.C1039Y). This mouse model displays AscAA, mitral valve disease, lung disease, and skeletal overgrowth - all hallmark symptoms of MFS (39). The MFS mouse model

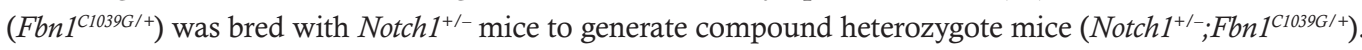
Echocardiograpic images of compound mutant mice and littermate controls were obtained at regular intervals between 1 and 5 months of age (Figure 1, data not shown). Notch1 ${ }^{+/-} ; F b n 1^{C 1039 G /+}$ mice displayed a significantly greater aortic diameter and a higher rate of dilation of the aortic sinus from 1 to 5 months of age in comparison with littermate controls (Figure 1, A-C, F, and G). Echocardiographic results were supported by microCT imaging that showed an increased aortic diameter in compound heterozygote mice in comparison with $F b n 1^{C 1039 G /+}$ mice at the aortic root, STJ, and ascending aorta. Compound mutant mice did not display BAV ( $n=4$, data not shown). Descending thoracic aortic diameters were normal across all genotypes (Figure 1, D and E, Supplemental Figure 1, and Supplemental Videos, 1-4; supplemental material available online with this article; https://doi.org/10.1172/jci.insight.91353DS1). Catheter-based blood pressure was measured, and no differences were observed between compound heterozygote and $\mathrm{Fbn} 1^{\mathrm{C} 1039 \mathrm{G} / \mathrm{+}}$ mice. Interestingly, Notch1 ${ }^{+/-}$mice had diastolic, systolic, and mean arterial pressures of 6-8 $\mathrm{mm} \mathrm{Hg}$ lower than WT (Supplemental Figure 2), which, if anything, should have a beneficial effect on the observed phenotype.

Increased aortic dilation was observed grossly (Figure 1, H-K) and accompanied by pathologic aneurysmal changes. As expected, the $F b n 1^{C 1039 G /+}$ aortas display hallmarks of aneurysm, including acellular space within the medial layer (Figure $1 \mathrm{~N}$, arrows), elastin degradation, and an increase in collagen when 
A

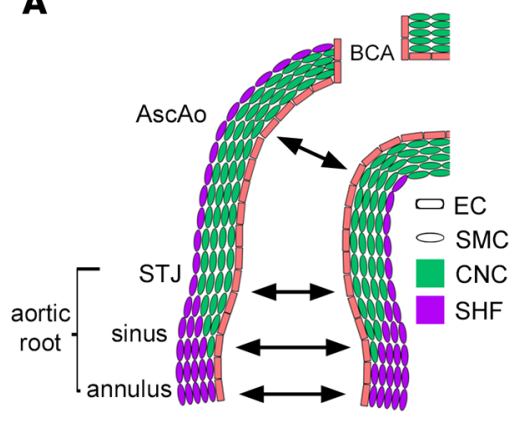

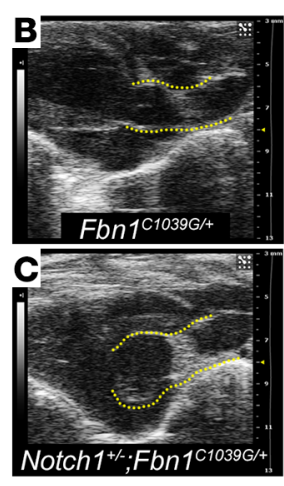

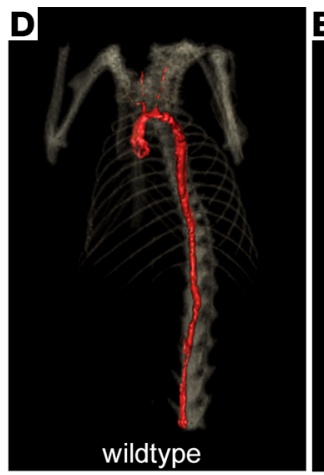

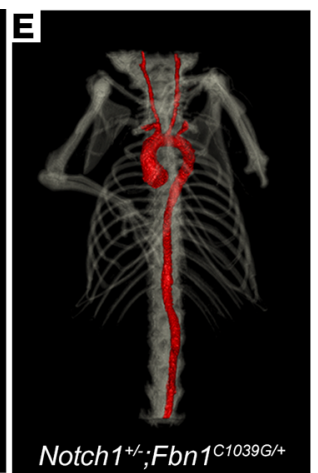

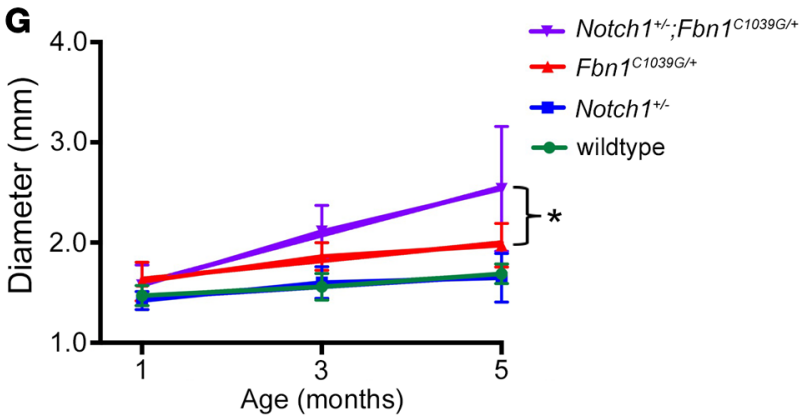

Age (months)
F

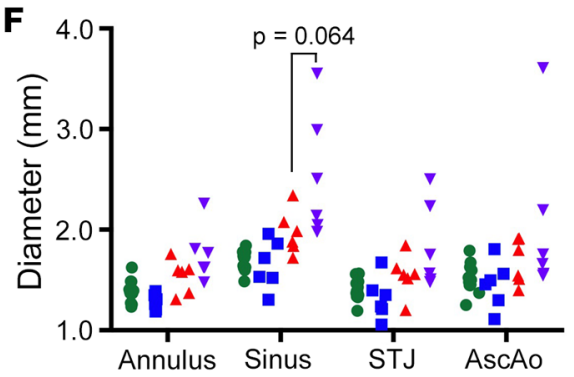

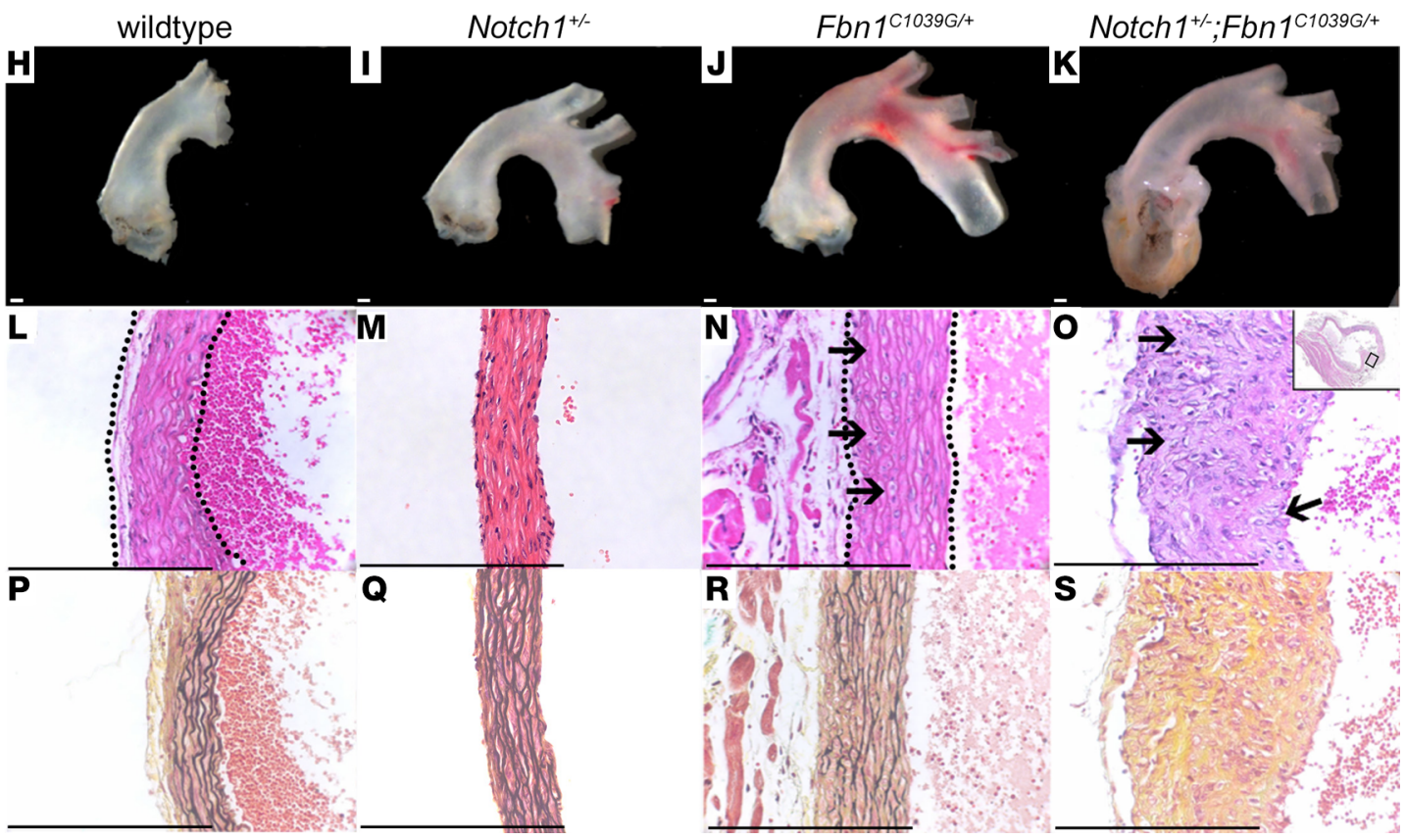

Figure 1. Ascending aortic aneurysm phenotype in Marfan syndrome mice is exacerbated by associated heterozygosity of Notch1. (A) Schematic of the thoracic aorta detailing the locations measured during echocardiographic analysis and showing the cell lineages that contribute to the smooth muscle cells. Purple (second heart field [SHF]), green (cardiac neural crest [CNC]). EC, endocardial cell. SMC, smooth muscle cell. (B and C) Represen-

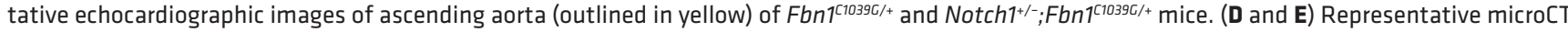
images of aorta of WT and Notch1+/-Fbn $7^{c 10396 /+}$ mice. (F and $\left.\mathbf{G}\right)$ Quantification of aortic diameter and growth rate demonstrating exacerbated disease in

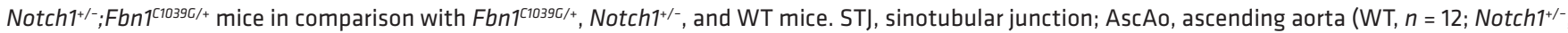

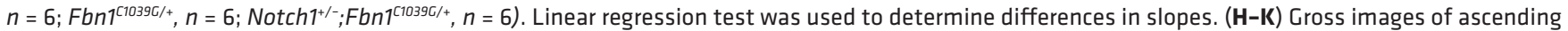

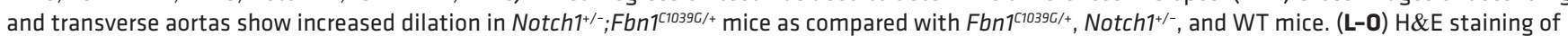

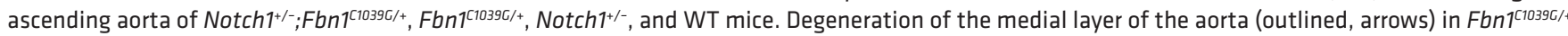
mice is noted, and it is substantially more severe in the Notch $7^{+/-}$; Fbn $7^{c 10396 /+}$ aortas. Section of whole aorta shown in $\mathbf{0}$, with box showing location of

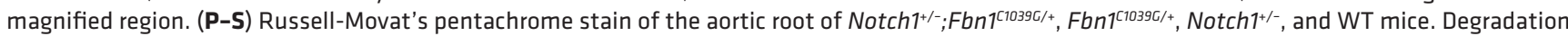
of elastin (black) and increased collagen deposition (yellow) in Fbn $7^{(10396 /+}$ aortas is found, along with increased collagen and complete regional loss of elastin in Notch1+/-; Fbn $7^{10396 /+}$ mice. ${ }^{*} P<0.05$. Scale bars: $200 \mu \mathrm{m}$. 
compared with WT and Notch1 ${ }^{+/-}$mice (Figure 1, L-N and P-R). Notch1 heterozygosity exacerbates this pathology with further increase in acellular space within the medal layer (Figure 1O), complete regional loss of elastin, and increased collagen deposition (Figure 1S). Furthermore, a small yet significant subset of the compound heterozygote mice succumbed to aortic rupture by 3 months of age (4/66), which was not observed in the Fbn1 ${ }^{C 1039 G /+}(0 / 63)$, Notch1 ${ }^{+/-}(0 / 51)$, or WT $(0 / 68)$ littermate controls $(P<0.05)$.

Loss of Notch1 in the SHF and not the endothelial cell lineage exacerbates AscAA in MFS mice. A series of compound mutant mice harboring a cell lineage-specific deletion of Notch1 and the Fbn $1^{\text {C1039G/+ }}$ mutation were generated in order to gain insight into the mechanism of Notch1 in AscAA (Supplemental Table 1). Notch1 is essential in endothelial cells for proper development of the vasculature (32) and in the endocardium for endocardial cushion formation and ventricular trabeculation $(41,42)$. Roles for endothelial Notch1 have also been described in adult cardiovascular disease, including valve calcification $(43,44)$. Therefore, we hypothesized that loss of Notch1 in endothelial cells was leading to the observed phenotype in the Notch1 ${ }^{+/-} ; F b n 1^{C 1039 G /+}$ mice. To test this, we utilized Tie2-Cre $e^{+}$mice to introduce heterozygosity of Notch1 into the endothelial cells of the MFS mice $\left(N o t c h 1^{+/ E C d e l} ; F b n 1^{C 1039 G /+}\right)(45)$. Although Notch1 is commonly found to function in endothelial cells in cardiovascular development and disease, the $N o t c h 1^{+/ E C d d l} ;$ Fbn $1^{\text {CI039G/+ }}$ mice did not recapitulate the phenotype of the $\mathrm{Notch1}^{+/-} ; \mathrm{Fbn} 1^{\mathrm{ClO} O 3 G /+}$ mice (Figure 2).

Several publications have demonstrated a less predominant role for Notch1 in SMC in the vasculature, including neointimal repair after injury (46-48). Therefore, we sought to determine if loss of SMC-specific Notch1 was the cause of the exacerbated phenotype observed in the $N o t c h 1^{+/-} ; F b n 1^{C 1039 G /+}$ mice. Heterozygous deletion of Notch1 using Myh11-Cre ${ }^{+/-}$mice demonstrated no evidence of AscAA, although a subtle increase in the aortic diameter at the level of the sinus (mean, $2.07 \mathrm{~mm} ; \mathrm{SD}, 0.11 \mathrm{~mm}$ ) compared with control littermates (mean, $1.97 \mathrm{~mm}$; SD, $0.11 \mathrm{~mm}$ ) was noted (Supplemental Figure 3) (49). Since the Myh11 promoter is only activated in mature SMCs, we tested whether Notch1 could be functioning in immature SMCs or SMC precursor cells during development of the ascending aorta.

SMCs of the ascending aorta are derived from 2 progenitor cell populations, the SHF and the CNC (Figure 1A). Mice with heterozygous deletion of Notch1 in each of these cell lineages, through the use of $\mathrm{Mef2C}-\mathrm{Cre}^{+}$and $\mathrm{Wnt1}-\mathrm{Cre}{ }^{+}$mice, in combination with a $\mathrm{Fbn} 1^{\mathrm{ClO3g} G /+}$ mutation were generated to determine if a single cell lineage could be responsible for the observed phenotype $(50,51)$. Interestingly, only loss of

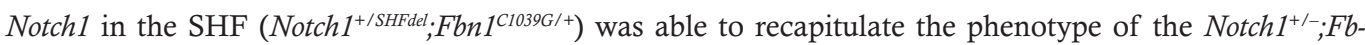
$n 1^{\text {C1039G/+ }}$ mice (Figure 3 and Supplemental Figure 3), which includes aortic dilation, loss of SMCs, regional loss of elastin, and increased collagen deposition in comparison with $N o t c h 1^{+/ f l} ; F b n 1^{C 1039 G /+}$ littermates. Finally, a subset of Notch1 $1^{+/ S H F d e l} ; F b n 1^{C 1039 G /+}$ mice succumbed to aortic rupture (2/13), which was not observed in $N o t c h 1^{+/ f l} ; F b n 1^{C 1039 G /+}$ littermates (0/11). The slight increased incidence in aortic rupture in the Notch1 ${ }^{+/ \text {SHFdel }} ; \mathrm{Fbn} 1^{\mathrm{C1039G/+}}$ mice, although not statistically significant, may be attributed to strain variation in the $\mathrm{Mef} 2 \mathrm{C}-\mathrm{Cr} e^{+}$mice.

We found additional evidence to support a role for Notch1 in SMC precursor cells by deleting Notch1 using the Myocardin-Cre (Myocd-Cre), which is expressed in SMC precursors and cardiomyocte progenitor cells

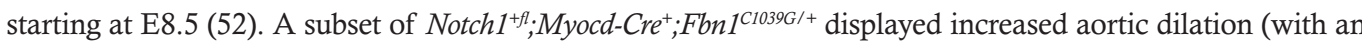

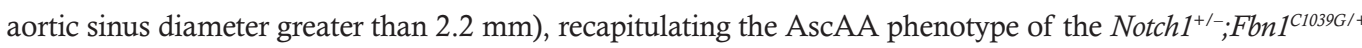
mice (Supplemental Figure 3). These findings indicate that loss of Notch1 in SMC precursors, but not mature SMCs, of the SHF predisposes them to AscAA, which reveals a potentially novel, developmental role for Notch1 in AscAA.

SHF-specific KO of Notch1 results in aortic arch anomalies and aortic dilation by 1 month of age. In order to study the effects of Notch1 in the SHF-derived SMCs, we wanted to make a more simplistic mouse model that was free of the potentially confounding Fbn1 mutation. Therefore, we generated mice with a homozygous deletion of Notch1 in the SHF cell lineage (Notch1 ${ }^{\text {SHF-KO}}$ ) to determine if this is sufficient to cause ascending aortic dilation. The resulting mice had $\sim 90 \%$ postnatal lethality (Supplemental Table 2 ), despite a normal gross appearance at E15.5 (Supplemental Figure 4, F and G). Histological sections of E15.5 hearts reveal cardiac outflow tract malformations in 3/4 embryos, including double-outlet right ventricle with ventricular septal defect and persistent truncus arteriosus (Supplemental Table 3 and Supplemental Figure 4, A-E). These mice also had structural anomalies of the aortic arch (4/4) characterized by tortuosity and abnormal branch placement (Supplemental Figure 5, E and F). These findings are consistent with previous publications where disruption of all Notch signaling (using dominant-negative allele of mastermind-like protein) or deletion Jagged1 (Jag1) in the SHF lineage led to cardiac outflow tract and aortic arch artery defects, and are likely due 

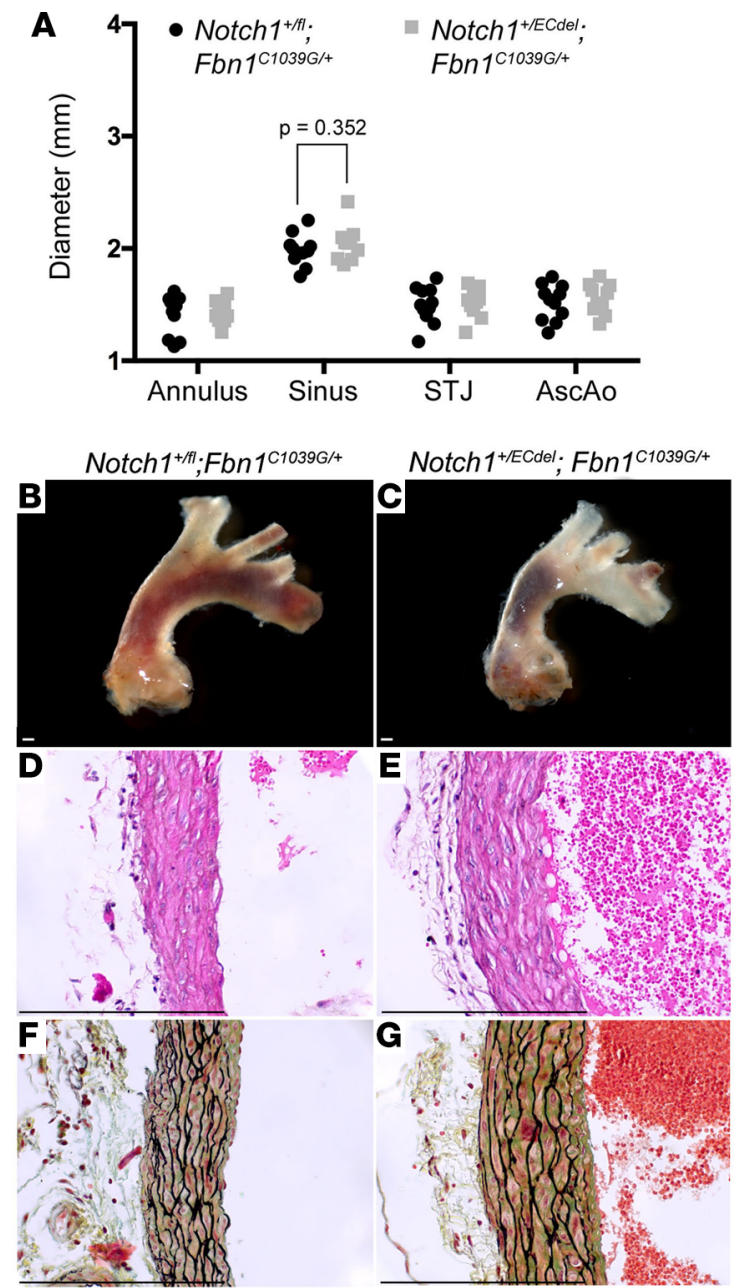

Figure 2. Endothelial Notch1 is not responsible for exacerbated aneurysm phenotype observed in Notch1+/-; Fbn $\mathbf{1}^{\mathbf{c 1 0 3 9 6 / +}}$ mice. Notch1 heterozygosity was introduced in endothelial and endothelial-derived cells with Tie2-Cre ${ }^{+/-}$mice (denoted $\mathrm{Notch}^{+/ / E C d e}$ ). (A) Quantification of echocardiographic analysis detailing aortic diameter at 4 locations in the thoracic aorta. No differences were observed between groups. ST], sinotubular junction; AscAo, ascending aorta (Notch1+/fl; Fbn $7^{10396 /+}, n=11 ;$ Notch1 $^{+/ E C d e l}$; Fbn $7^{c 10396 /+}, n=10$ ). (B and C) Representative gross images of aortas from Notch $1^{+/ f f}$; Fbn $1^{\text {C1039C/+ }}$ and Notch $1^{+/ E C d e l} ;$ Fbn $1^{C 10396 /+}$ mice ( $n=3 /$ genotype). Differences were not observed in medial integrity with $\mathrm{H} \& \mathrm{E}$ stain ( $\mathbf{D}$ and $\mathbf{E}$ ) or with Russell-Movat's pentachrome (F and $\mathbf{G})$ ( $n=3 /$ genotype). Scale bars: $200 \mu \mathrm{m}$.

to aberrant neural crest cell migration $(33,35)$. Our data indicate that Notch1 is the receptor via which the ligand Jag1 is signaling through during outflow tract development. This is further supported by immunofluorescent staining in WT embryos at E11.5 displaying coexpression of NICD1 and Jag1 around the aortic sac and in the aorticopulmonary septum (Supplemental Figure 4, H and I). Surviving Notch1 $1^{\text {SHF-KO }}$ mice appeared to have effacement of the STJ, an early indicator of aortopathy, by 1-2 months of age by echocardiogram (Supplemental Figure 5, A-D). Due to variations in pup sizes by litter at this time point, a ratio of STJ diameter to the sinus diameter was calculated and demonstrated statistically significant STJ effacement (Supplemental Figure 5B).

Notch $1^{+/-}$mice on 129 SV background develop AscAAs. Mouse strain differences are known to contribute to phenotypic variability, and accordingly, we generated $\mathrm{Notch}^{+/-}$mice in a predominant $129 \mathrm{~S} 6$ background (>98\%) by backcrossing for 5 generations (Notch1.129S6). By 2 months of age, mild aortic root dilation was observed in a subset of the Notch1.129S6 $6^{+1-}$ mice in comparison with WT littermates (Figure 4A). Time course studies

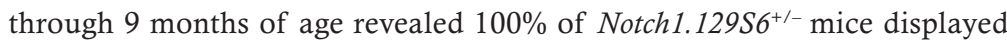
aortic root dilation in comparison with WT littermates (Figure 4, B-D). Histologic examination of the Notch1.129S6 $6^{+/}$aorta at 9 months of age revealed localized thinning of the aortic wall (Figure 4, E and F). Within the thinned region, SMCs had abnormal morphology with dysmorphic, rounded nuclei and a reduction in elastic laminae (Figure 4, G-J), demonstrating that heterozygous deletion of Notch1 causes AscAA in mice. Of note, aortic root dilation was not observed in Notch $^{+/-}$mice in a mixed background strain (Figure 1), and Notch1.129S6 ${ }^{+/-}$mice did not have BAV ( $n=6$, data not shown).

Loss of Notch1 results in increased aneurysm-related gene expression in aortic root. RNA-seq was performed on proximal and distal ascending aortic tissue from Notch1.129S6 ${ }^{+/-}$mice and WT littermates at 2 months of age (Figure 5A). Transcriptome analysis was utilized to better understand why the dilation was localized to the aortic root. Hierarchical cluster analysis grouped these samples based on location first and then genotype, and showed that cells of the proximal and distal ascending aorta have distinct gene expression patterns in vivo (Figure 5, B and C). Next, we examined alterations in cell signaling due to Notch1 heterozygosity, focusing on the aortic root, which is predominately of the SHF cell lineage (53). A heatmap of hierarchical clustering shows differential gene expression by region, and a volcano plot highlights the differential gene expression in Notch1.129S ${ }^{+/-}$vs. WT proximal aortas (Figure 5, C and D). Functional annotation of significantly differentially expressed genes — utilizing Database for Annotation, Visualization and Integrated Discovery (DAVID) — identified changes in gene ontology (GO) terms associated with focal adhesion, adherens junction, ECM, and cell cycle regulation indicating gene expression changes associated with AscAA (Figure 5E). The top 10 most significantly different GO terms are represented as a circle graph in Figure 6A and highlight the overall upregulation in genes associated with each term. Additionally, the chord graph in Figure 6B shows the association of 18 genes with the top $8 \mathrm{GO}$ terms. Interestingly, these results are similar to gene expression profiling of human TAA tissues where focal adhesion and adherens junction pathways were implicated $(54,55)$. Accordingly, the observed differences in gene expression in the Notch1.129S6 $6^{+/}$mice demonstrate molecular changes consistent with the development of AscAA. 

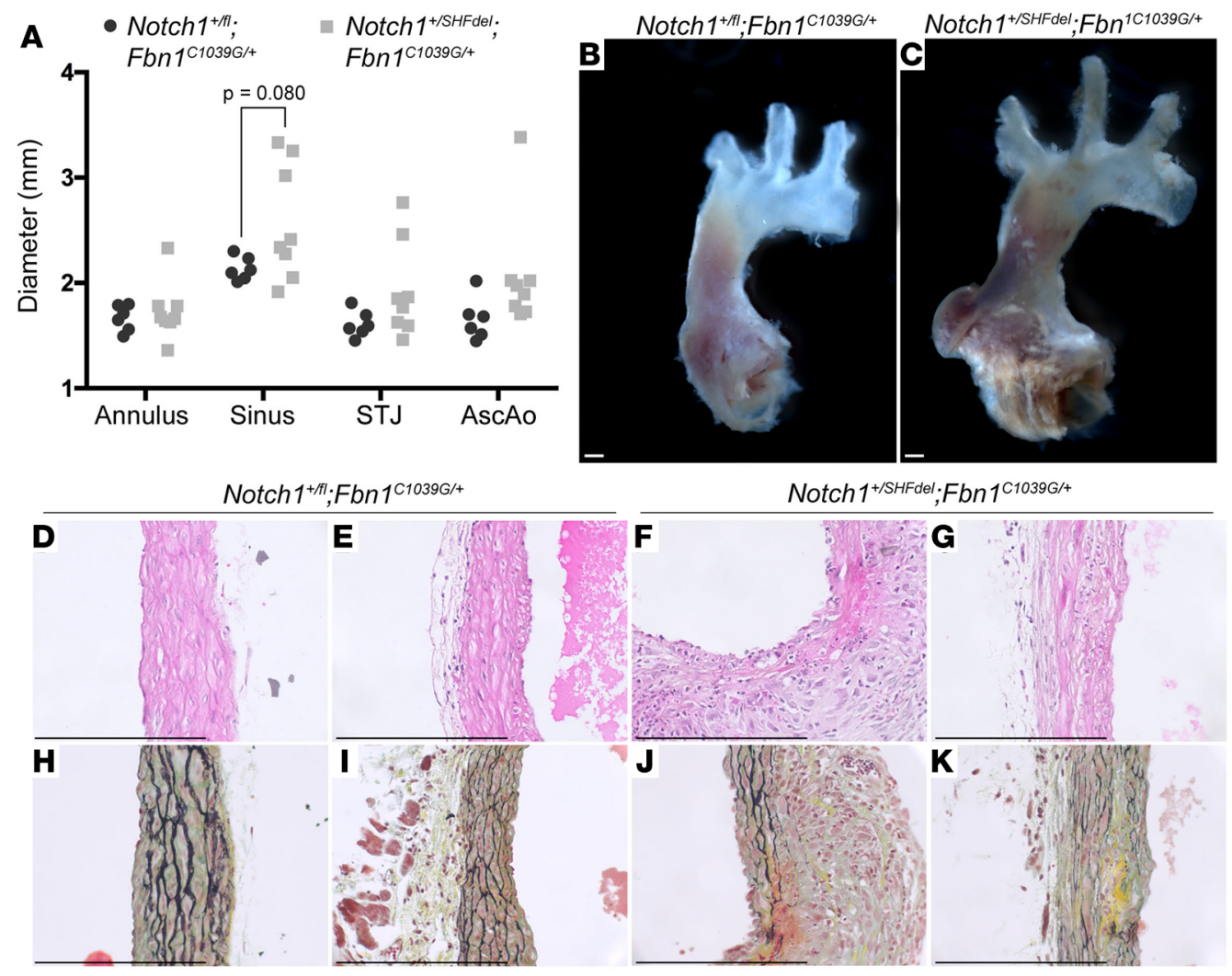

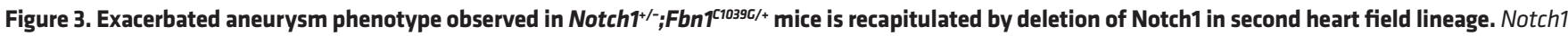
heterozygosity was introduced in second heart field-derived cells with Mef2C-Cre+/- mice (denoted Notch $1^{+/ S H F d e l}$ ). (A) Measurements of aortic diameter by

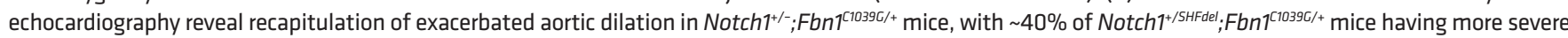

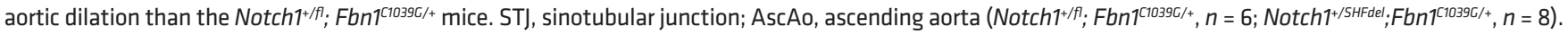

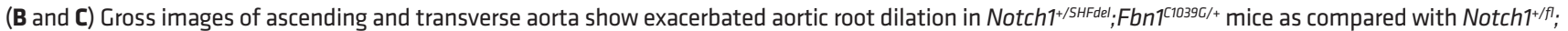
Fbn $7^{10396 /+}$ mice. H\&E stain reveals substantial medial degeneration (D-C), accompanied by regional loss of elastin (black) and increased collagen deposition (yellow), as shown by Russell-Movat's pentachrome staining (H-K) ( $n=3 /$ genotype). Scale bars: $200 \mu \mathrm{m}$.

\section{Discussion}

Mutations in NOTCH1 are associated with BAV in humans, but the link between Notch1 and BAV-associated AscAA has not been well studied. Here, we have utilized several murine models to describe a potentially novel role for Notch1 in the development of AscAA and have shown that loss of Notch1 in the SHF contributes to AscAA. We found that haploinsufficiency of Notch1 exacerbates the AscAA phenotype found in $F b n 1^{\mathrm{Cl039G/+}}$ mice, and this more severe phenotype is the result of heterozygous deletion of Notch 1 in SHF lineage. While we did not examine $N o t c h 1^{+/-} ; F b n 1^{C 1039 G /+}$ mice for BAV, Notch $1^{+/-}$mice were previously shown to have a much lower incidence of BAV than the incidence of AscAA observed in this study (34). In addition, we demonstrate the requirement of Notch1 in the SHF lineage for proper development of the cardiac outflow tract. Furthermore, we describe a Notch1 haploinsufficient mouse model of AscAA that is independent of BAV and presents with aortic root dilation, abnormal SMC morphology, and a decrease in elastic laminae, along with associated molecular changes.

Notch1 is expressed in the developing cardiac outflow tract, and mutations in NOTCH1 are known to cause aortic valve malformations in humans and mice. Previous studies have demonstrated the requirement of Notch1 in endothelial and endothelial-derived cells for proper development of the aortic and pulmonary valves. The finding that heterozygous deletion of Notch1 in SHF-derived cells causes AscAA independent of aortic valve disease is surprising. In addition, our data suggest that Notch1 plays a role in SMC precursors, as supported by our findings using the Mef2C-Cre and Myocd-Cre mice. As lineage-specific deletions are a critical portion of this work, it is important to note alternate interpretations of these results. Myocd is a transcriptional coactivator of Serum Response Factor and has a critical role in SMC differentiation and contraction. The Myocd-Cre mouse was created through excision of exon1 of Myocd (52); 

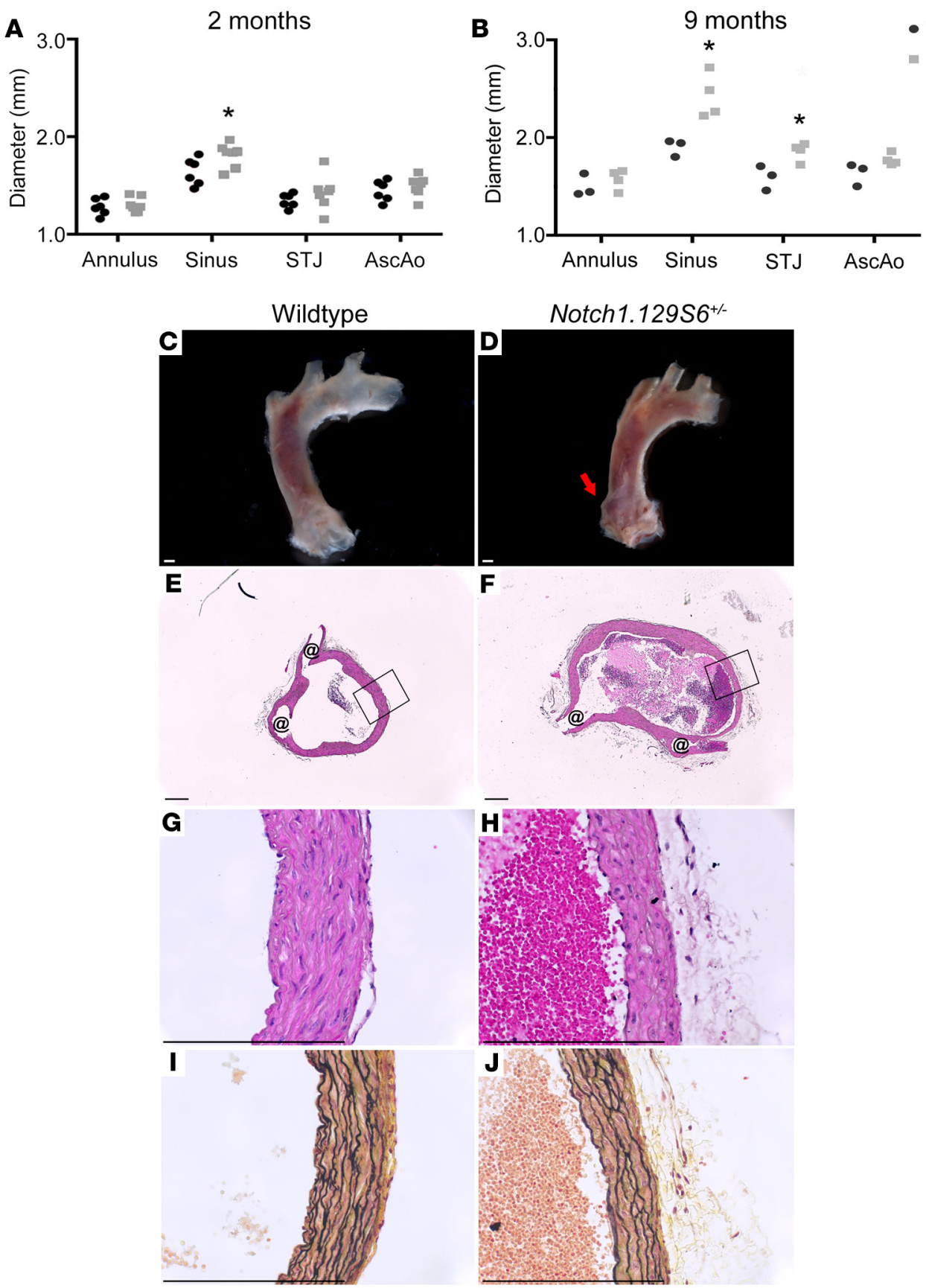

Figure 4. Notch1 heterozygosity in the 12956 background strain causes aortic root dilation. Notch1+/- mice were backcrossed 5 generations into a 12956 background (Notch1.129A6+/-) and investigated for evidence of aortopathy. (A and B) Quantification of thoracic aortic diameter at 4 sites in Notch1.129S6 ${ }^{+/-}$ mice and WT littermates (2 months: WT, $n=6$; Notch1.129S6 ${ }^{+/-}, n=7 ; 9$ months: WT, $n=3$; Notch1.129S6 ${ }^{+/}, n=4$ ). ST), sinotubular junction; AscAo, ascending aorta. (C and D) Gross images of ascending and transverse aorta displaying aortic root dilation (arrow) in Notch1.129S6 ${ }^{+-}$mice at 9 months of age as compared with WT littermate. (E and F) H\&E-stained aortas show dilation and thinning of aortic wall. (G and $\mathbf{H})$ Magnified region (represented by box in $\mathbf{E}$ and $\mathbf{F}$ ) of H\&E-stained aorta shows changes in smooth muscle cell morphology with dysmorphic nuclei, and (I and J) Russell-Movat's pentachrome staining reveals a reduction in elastic laminae in Notch1.129S6+/- mice as compared with WT mice. @, indicates left and right coronary arteries.

therefore, it is possible that it functions as a hypomorph. If Myocd is not fully expressed, the Notch1 ${ }^{+/ f \text {; }}$ Myocd-Cre $; F b n 1^{1033 G^{\prime} /+}$ mouse may have aortic dilation due to a combination of Myocd deficiency and the Fbn $1^{C 1039 /+}$ mutation. However, the SHF-specific deletion of Notch1 support our conclusions that it is Notch1 in SMC precursor cells. In addition to SMCs, endothelial cells and cardiomyocytes are also derived from the SHF. We demonstrated that endothelial Notch1 does not contribute to AscAA, but we have not ruled out that loss of Notch1 in both endothelial and SMC precursors cause the observed phenotype. Based on our 


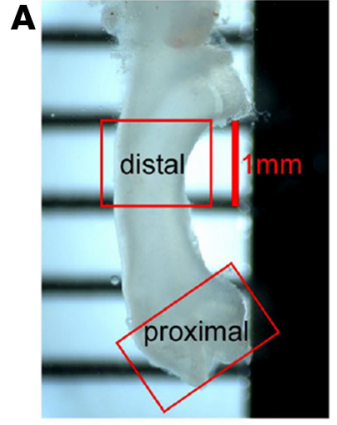

B

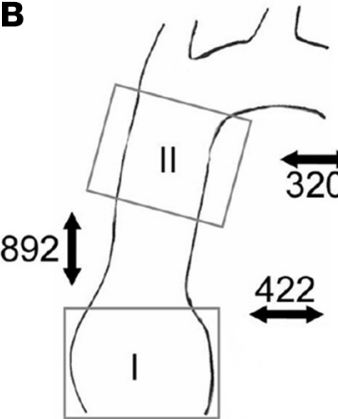

wildtype

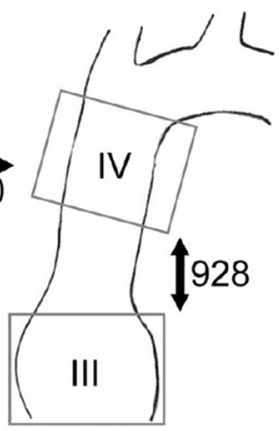

Notch1.129S6 $6^{+/}$

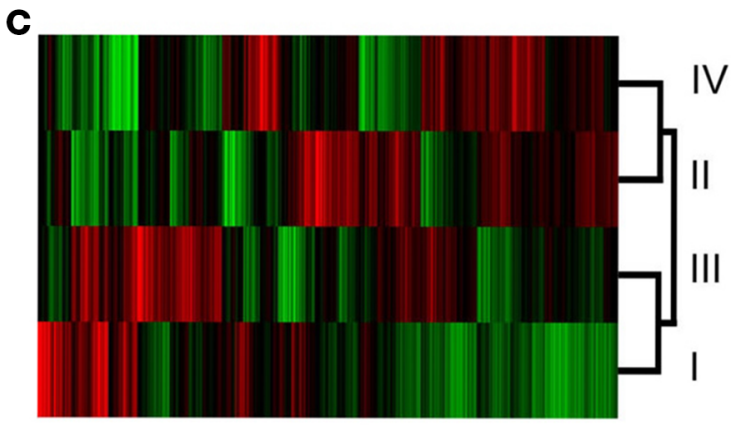

D Differential Gene Expression in Notch1.129S ${ }^{+/}$vs. Wildtype Proximal Aortas

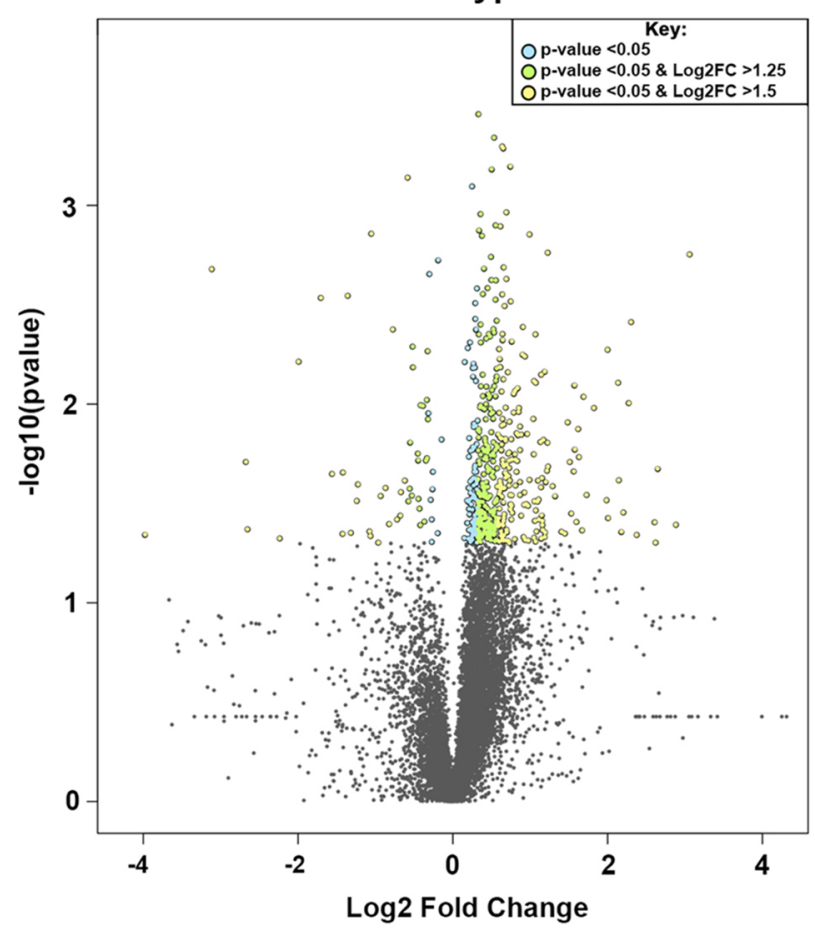

$\mathbf{E}$

Top 20 Gene Ontology Terms: Notch1.129S ${ }^{+/}$vs. Wildtype Proximal Aortas

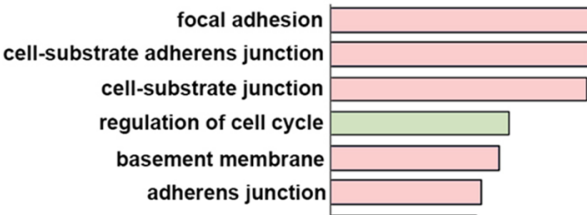

extracellular matrix component anchoring junction regulation of cell morphogenesis: differentiation regulation of anatomical structure morphogenesis nucleis acid templated transcription extracellular matrix regulation of cell cycle process regulation of RNA metabolic process regulation of transcription regulation of nucleic acid-templated transcription epithelial to mesenchymal transition RNA biosythetic process nucleoplasm

RNA pol II carboxy-terminal domain kinase activity

Key:

Biological Process

$\square$ Cellular Component

$1 \quad 0.01 \underset{p}{0.0001} \quad 0.00001$

Figure 5. RNA sequencing reveals gene expression changes in Notch1.12956/- aortic root. (A) Diagram showing 1 mm section of proximal (aortic root) and distal ascending aorta from 2-month-old mice that was used for RNA-seq ( $n=3$ /group). (B) Summary of differentially expressed genes that were identified with a fold change of \pm 1.5 and determined to have a $P<0.05$ by Tukey test. (C) Heatmap generated with the log ${ }_{2}$-transformed RPKM values of the 522 ANOVA $P<0.05$. Hierarchical clustering indicates samples associate strongest by location. (D) Volcano plot representing the differential gene expression

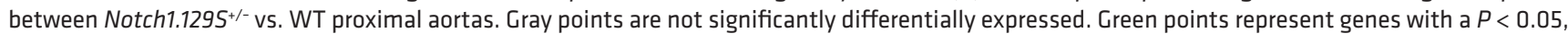
blue points represent genes with a $P<0.05$ and a $\log _{2}$ fold change $>1.25$, and yellow points represent genes with a $P<0.05$ and a log fold change $>1.5$. (E) Top 20 gene ontology terms enriched within the significantly differentially expressed genes between the Notch1.1295 ${ }^{+/-}$vs. WT proximal aortas. Terms were identified through analysis with the Database for Annotation, Innovation and Integrated Discovery (DAVID). Green bars represent GO-FAT biological processes, pink bars represent GO-FAT cellular component, and blue bars represent GO-FAT molecular function.

cell-specific deletions and SHF lineage tracing studies, our data support the conclusion that loss of Notch1 in SMC precursors from the SHF contributes to aortopathy of the ascending aorta. It is important to note the limitation that the lineage studies were performed in mice with a mixed background; therefore, we can not definitively conclude that the Notch1.129S6 ${ }^{+/-}$mice have a decrease in SHF-derived SMCs in the aortic root.

While loss of Notch1 in endothelial cells contributes to the development of BAV, these studies provide molecular and cellular insights into the mechanisms by which loss of Notch1 in the SHF lineage-derived cells contributes to AscAA. As discussed, several progenitor cell populations differentiate into SMCs to form different segments of the thoracic aorta (reviewed in ref. 56). Cells of the SHF lineage primarily differentiate into SMCs in the aortic root (57). CNC cells comprise the SMCs in the ascending and transverse aorta, while somatic mesoderm cells form the descending thoracic aortic SMCs (Figure 1A). It has been proposed that, 
A
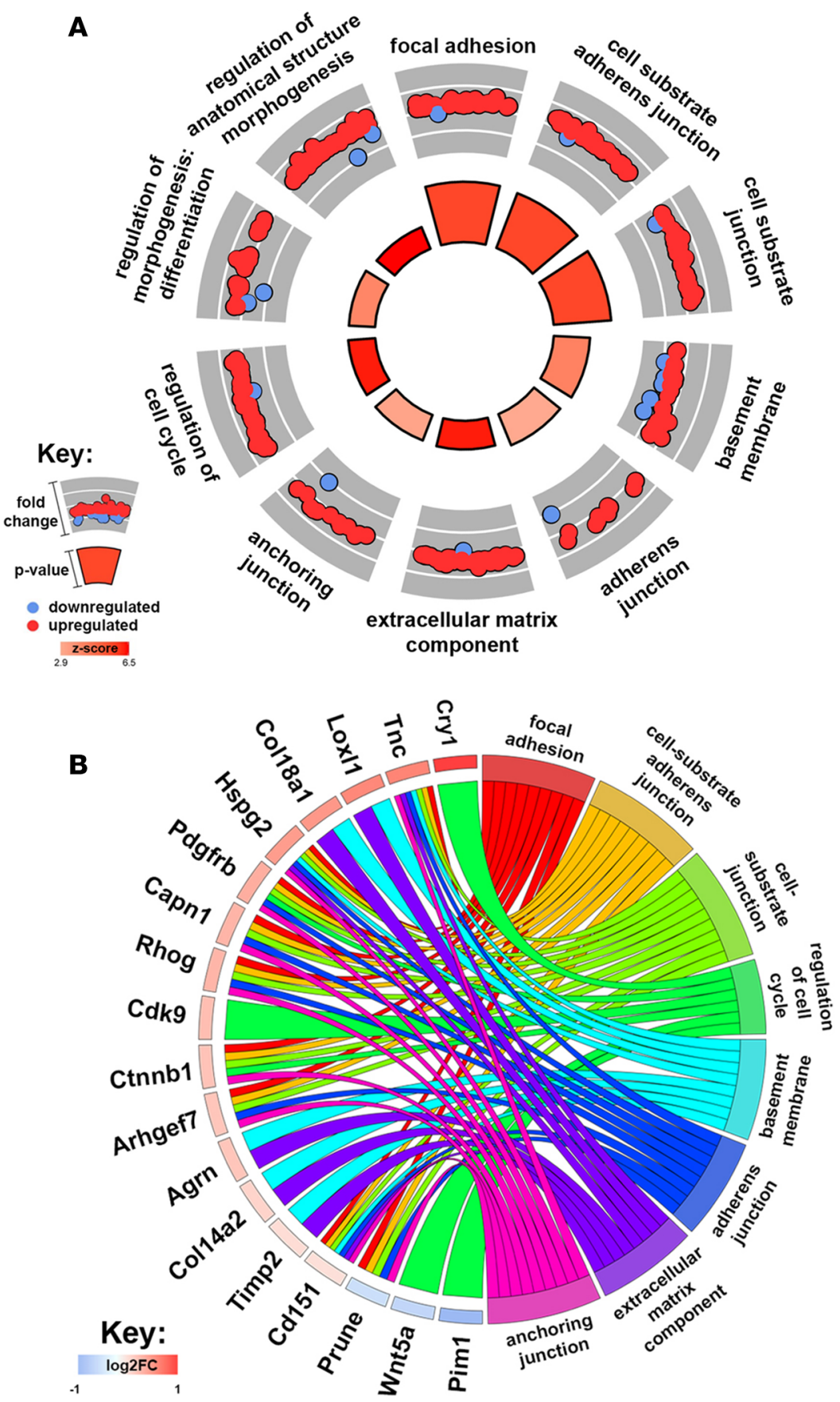

Figure 6. Gene expression profiling of Notch1.12956 ${ }^{+/}$ aortic root consistent with aortopathy. (A) Circle plot representing gene annotation and enrichment of the top $10 \mathrm{GO}$ terms. The outer circle scatter plots represent the differential gene expression within each term, with red points representing upregulated genes and blue points representing downregulated genes. The inner circle is colored based on $Z$ score, which is a measure for predicting a bias in gene regulation, with blue predicting a decrease in the pathway and red predicting an increase. Additionally, the inner circle is sized based on $P$ value, with the larger size correlating to a more significant $P$ value. (B) Chord graph representing 18 differentially expressed genes between the Notch1.1295//- vs. WT proximal aortas and the association of these genes to the corresponding top 8 GO terms. The gene name color code represents the $\log _{2}$ fold change, with blue corresponding to downregulation and red corresponding to upregulation.

although the resulting SMCs are phenotypically similar, in vivo signaling mechanisms differ according to cell lineage (58-61).

A potential mechanism for the observed AscAA phenotype with loss of Notch1 in the SHF lineage could be the differences in the contribution from each of the cell lineages in the aorta of mutant Notch1+1mice. We performed preliminary lineage tracing experiments utilizing ROSA26mT/mG and $R 26 R$ mice, which allow for tracing of SHF-derived cells after breeding to $\mathrm{Mef2C}-\mathrm{Cre}^{+}$mice through expression of green fluorescent protein or $\beta$-galactosidase in cells that are derived from Cre recombinase-expressing cells (62). These studies suggest a reduction in SHF-derived SMCs in the aortic root of postnatal day $8 \mathrm{Notch}^{+ \text {SHFFel }} ;$ ROSA26 $6^{\text {mT/mG }}$ or $\mathrm{Notch}^{+/ \text {SHFdel }}$, $R 26 R$ mice, but inconsistency in our results with either methodology preclude definitive conclusions (Supplemental Figure 6). Additional in vivo experiments using transgenic mice, where cells of the MEF2c-AHF and Notch1 lineages are both genetically labeled, are needed to determine if this is the underlying mechanism $(63,64)$. Additionally, it is unknown if the mechanism for the AscAA phenotypes observed in the $\mathrm{Notch}^{+/-}$; Fbn $1^{\mathrm{Cl} 1039 \mathrm{G} / \mathrm{+}}$ model and the Notch1.129S6 $6^{+/-}$model is the same, and this is a limitation of this study.

Molecular characterization of the ascending aorta of Notch1.129S6 $6^{+-}$mice using RNA-seq identified dysregulation of many pathways associated with AscAA. Within this list, 2 genes - tenascin C (Tnc) and lysyl-oxidase-like 1 (Loxl1) - have previously been shown to play roles in the pathogenesis of aortic aneurysms. Tnc encodes an ECM protein that is highly expressed in development but is only present in adult humans and mice with vascular disease (reviewed in ref. 65). Tnc has roles in SMC proliferation and migration during neointimal formation $(66,67)$. It has also been shown to be upregulated in tissue samples from patients with AscAA and to protect mice from aortic dissection in a mouse model of abdominal aortic aneurysm (68-71). Loxl1 is a member of the lysyl oxidase family, which facilitates collagen and elastin crosslinking and is protective against abdominal aortic aneurysm progression (72). Busnadiego et al. found increased LOXL1 expression in aortic tissue samples from MFS patients and in the $\mathrm{Fbn}^{\mathrm{C} 1039 \mathrm{G} / \mathrm{+}}$ mice, correlating with increased collagen deposition (73). Additionally, chemical inhibition of lysyl oxidases resulted in exacerbation of the 
AscAA in $\mathrm{Fbn}^{\mathrm{ClO} 103 \mathrm{G} /+}$ mice, again suggesting a protective role for this gene (73). The findings in our RNA-seq analysis are interesting in that they were performed at 2 months of age, when there was only mild evidence of aneurysmal disease, and it will be interesting to examine earlier time points for molecular differences in Notch1.129S6 ${ }^{+/-}$mice. While the clinical and translational significance of our RNA-seq findings are unclear, this new mouse model of AscAA may serve as an important disease model to identify novel biomarkers for the development of AscAA. We also examined the expression of the Notch1 intracellular domain in normal and diseased aortic tissue from humans and found expression levels correlated with disease severity, suggesting that it too may serve as a biomarker (Supplemental Figure 7). These findings are similar to those reported by Zou et al., where Notch1 expression is increased in descending TAA tissues, although this appears to be driven by expression in non-SMC populations (74). The clinical significance of Notch1 levels in humans with abdominal aortic aneurysms has been examined; it remains to be studied in patients with AscAA (75).

It is worth investigating whether other genes that have been associated with BAV also have an independent role in contributing to aortic root SMCs or AscAA. Mutations in GATA5 have been linked to BAV in humans $(76,77)$, and while mice with genetic deletion of Gata5 display BAV, AscAA was not reported (78). Furthermore, it would be interesting to determine if genes that are associated with AscAA, such as FBN1 (3-6) and TGFBR1/2 (7), have a role in aortic development that predisposes to the development of AscAA. Our data warrant investigation into potential effects of Fbn1 mutations on the cell lineage contributions to the SMCs lining the ascending aorta. While much attention has focused on the TGF- $\beta$ signaling pathway, it is possible that developmental anomalies in conjunction with increased TGF- $\beta$ signaling could be responsible for the AscAA observed in this population.

This work paves the way for an alternative discussion of BAV-associated aortopathy, which affects approximately $40 \%$ of the BAV population and is observed in both the proximal and distal ascending aorta $(79,80)$. BAV-associated aortopathy has been attributed to altered hemodynamics and genetics. We propose that a subset of the BAV-associated aortopathy population has developmental origins that are tied to abnormal aortic valve development, making them more susceptible to aortopathy with the introduction of altered hemodynamics. Additional investigation is required to identify BAV disease-causing genes that function in only endothelial cells or also in SMC precursors of the developing cardiac outflow tract, as this may lead to important genotype-phenotype insights for this patient population.

\section{Methods}

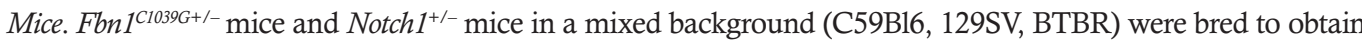
Notch $1^{+/-F b n} 1^{\mathrm{C1039G/+}}$ mice and littermate controls and were genotyped with primers specified by The Jackson Laboratory. For lineage-specific deletions of Notch1 (using Tie2-Cre [ref. 45], Myh11-Cre [ref. 49], Myocardin-Cre [ref. 52], Mef2C-Cre [ref. 50], and Wnt1-Cre [ref. 51]) in a MFS mouse background, Fbn ${ }^{\mathrm{ClO} 39 \mathrm{G} /{ }^{+}} ; \mathrm{Cre}^{+/-}$male mice were bred to homozygous female mice with LoxP sites flanking exon1 of Notch1 (Notch $1^{f / f f}$ ) (81) to obtain Notch $1^{f /+}$;

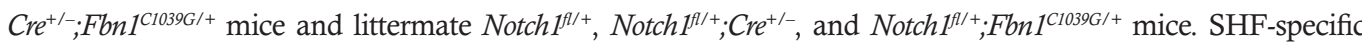
Notch1-KO mice (Notch ${ }^{\text {SHF-KO}}$ ) were obtained by breeding $N o t c h 1^{f /+} ; M e f 2 C-C r e^{+/-}$males to Notch $1^{f l f l}$ females. Lineage tracing of SHF cells in $\mathrm{Notch}^{+/-}$mice was performed by breeding $\mathrm{Notch}^{+/-} ; \mathrm{Mef2C}$-Cre $\mathrm{C}^{+/-}$males to $R O S A 26^{m T / m G}$ or $R 26 R$ (82) females. The resulting pups were euthanized at postnatal day 8. Notch1.129S6 ${ }^{+/-}$mice were created by backcrossing Notch $1^{+/-}$mice 5 generations to the $129 \mathrm{~S} 6$ mouse strain (Taconic Biosciences).

Echocardiography. Transthoracic echocardiography was performed using a VEVO 2100 Ultrasound System as previously described. Mice were sedated briefly with $3 \%$ isoflurane and then titrated to $1 \%-2 \%$ isoflurane to maintain heart rate between 400 and $500 \mathrm{bpm}$. Aortic diameters were measured from parasternal long axis B-mode images during systole at the aortic annulus, sinus, STJ, and ascending aorta (distal to the pulmonary trunk). The average of 3 measurements at each location was plotted with Prism.

MicroCT imaging and reconstruction. Mice were anesthetized briefly with 3\% isoflurane (Baxter Healthcare Corporation) to effect, and then titrated to $1 \%-1.5 \%$ isoflurane, which was maintained for the duration of imaging. AuroVist 15 nm: Gold Blood Pool x-ray Contrast Agent (100 $\mu 1$, Nanoprobes) was administered via tail vein injection. Whole-body MicroCT was performed with the GE eXplore Locus (GE Healthcare) at a spatial resolution of $45 \mu \mathrm{m}$. The reconstructed slices were output in the CT manufacturer's raw format (DICOM), and the reconstructed images were viewed and analyzed using the Vitrea Enterprise Suite Software 6.7.0 (Vital, A Toshiba Medical Systems Company). The DICOM images were initially reconstructed into an axial, coronal, and sagittal planes to allow identification of the aorta. Using 2 orthogonal planes, a cross section of the aorta at each location was measured. 
Blood pressure. Mice ( $n=4$ /genotype) were anesthetized using isoflurane anesthesia vaporized with $100 \%$ $\mathrm{O}_{2}$ (4\% induction, $2 \%$ maintenance for the duration of the experiment). A midline incision was made on the neck, and the right common carotid artery was isolated. A mikro-tip pressure catheter (1.2F, SciSense, Transonic Systems Inc.) connected to a PowerLab data acquisition system (AD Instruments) was inserted into the carotid, advanced into the aorta, and secured with 5-0 silk. Blood pressures were continuously recorded using LabChart 7 software (AD Instruments). After instrumentation, mice were allowed to equilibrate for 30 minutes prior to making average blood pressure measurements over 3 minutes of recording time.

Tissue fixation and histology. Experimental animals were euthanized, and mouse hearts were gravity perfused with $\sim 1 \mathrm{ml}$ of $10 \%$ formalin into the left ventricle. Tissue was embedded in paraffin, and serial sections were stained with hematoxylin (MilliporeSigma, GHS316) and eosin (MilliporeSigma, HT110316) and Russell Movat pentachrome (American MasterTech, KTRMP). Proximal aortic sections were obtained within the root, and distal aortic sections were obtained by sectioning 1,200 $\mu \mathrm{m}$ past the coronary arteries.

Immunostaining. Immunofluorescence for NICD1 (Abcam, ab8925, 1:200), Jag1 (Santa Cruz Biotechnology Inc., sc-6011, 1:200), GFP (Abcam, ab290, 1:1000), and aSMA (MilliporeSigma, A2574,1:1000) was performed overnight at $4^{\circ} \mathrm{C}$. Secondary antibodies were used at a dilution of 1:400 (goat anti-rabbit Alexa Fluor 488, Invitrogen, A-11008) for NICD; at dilution of 1:400 (donkey anti-goat Alexa Fluor 594, Invitrogen, A-11058) for Jag1; at a dilution of 1:500 (donkey anti-rabbit Alexa Fluor 488, Invitrogen, A-21206) for GFP; and at a dilution of 1:1,000 (donkey anti-mouse Alexa Fluor 568, Invitrogen, A-10037) for $\alpha$ SMA. Negative controls for all immunostaining was performed without primary antibody.

$\beta$-Galactosidase activity assay. Lineage tracing of SHF cells was performed with $R 26 R$ mice. $\beta$-Galactosidase activity was assessed following a protocol published by Cold Spring Harbor Laboratory.

$R N A$-seq. Proximal and distal aortic tissue samples were collected from $3 \mathrm{WT}$ and 3 Notch1.129S6 $6^{+/}$ mice at 2 months of age. Tissue was collected in Trizol (Ambion), snap frozen, and homogenized using a TissueLyserII (Qiagen). RNA was isolated using a combination of Trizol and Total RNA Purification Kit (Norgen Biotek). Twelve total RNA samples from mouse aorta tissues ( $n=3$ of each group) were submitted to Ocean Ridge Biosciences for mRNA-seq. The RNA was digested with RNase free DNase I (Epicentre, D9905K) and repurified on RNeasy MinElute columns (Qiagen, 74204) using an alternative high-ethanol binding condition to preserve low molecular weight (LMW) RNAs. The newly prepared digested total RNA samples were then quantified by Quant-iT RiboGreen RNA fluorometric assay (Thermo Fisher Scientific, R11491) and assessed for quality by Agilent2100 Bioanalyzer RNA 6000 Pico Assay (Agilent Technologies, 5067-1513).

Amplified cDNA was prepared from a total of $10 \mathrm{ng}$ of the digested total RNA samples using the NuGen Ovation RNA-Seq System v2 (NuGen, 7102-A01) and fragmented using NEBNext dsDNA Fragmentase (New England Biolabs, M0348L). Template DNA molecules suitable for cluster generation were then prepared from the fragmented cDNA using the Illumina TruSeq DNA Nano HT Library Preparation Kit (Illumina Inc., FC-121-4003). The quality and size distribution of the amplified cDNA libraries were determined by chip-based capillary electrophoresis using an Agilent 2100 Bioanalyzer DNA 1000 chip assay (Agilent Technologies, 5067-1504). The libraries were then quantified using the KAPA Library Quantification Kit (Kapa Biosystems).

The libraries were pooled at equimolar concentrations and diluted prior to loading onto the flow cell of the Illumina cBot cluster station. The libraries were extended and bridge amplified to create sequence clusters using the Illumina HiSeq PE Cluster Kit v4 and sequenced on an Illumina HiSeq Flow Cell v4 with 50-bp paired-end reads plus dual-index read using the Illumina HiSeq SBS Kit v4. Real-time image analysis and base calling were performed on the HiSeq2500 using the HiSeq Sequencing Control Software version 2.2.68.

The raw FASTQ files were split into files containing $4 \times 10^{6}$ reads and checked for quality using FASTX toolbox. Based on the quality results, the reads were filtered (removing sequences that did not pass Illumina's quality filter) and trimmed (3 nucleotides at the left end of R1 and -3 nucleotides off the left end of R2).

Sequence alignment to the mm10 mouse reference genome was performed using TopHat v2.1.0 (83) with fr-unstranded as the library type. The mate-inner-distance and mate-std-dev settings were determined for each sample by nongapped alignment of a portion of the reads to the mouse mRNA reference using Bowtie2 (84). All other TopHat settings were left as default.

Exon- and gene-level read counts were calculated using the easyRNASeq function of the easyRNASeq $\mathrm{R}$ package (85) version 2.4.7 running on $\mathrm{R}$ version 3.2.2. For gene-level read counts, the summarization method used was GeneModels. For annotation, an R data (RDA) file was generated from the Ensem- 
b1 Mouse Release 83 gene transfer format (GTF) file using the annotation file generation function of easyRNASeq. The RNA-seq data has been deposited on NCBI Gene Expression Omnibus (GEO, accession number GSE 104365).

$R N A$-seq data analysis. A heatmap was generated utilizing the $\log _{2}$-transformed reads per kilobase per million (RPKM) data for detectable mouse genes, and hierarchical clustering analysis was performed by Cluster 3.0 software (86). Genes were median centered prior to hierarchical clustering, and hierarchical clustering was conducted using centered correlation as the similarity metric and average linkage as the clustering method.

Functional annotation was performed on differentially expressed genes with a $P<0.05$ with the DAVID version 6.8. (87) GO-FAT terms were utilized, which filter out broad GO categories based on a measured specificity of each term. GO-term analysis was visualized with GOplot 1.0.2. (88). Bubble plots were generated with all GO-FAT terms utilizing GOBubble. The circle plot was generated using GOCircle, utilizing the top 10 most significant GO-FAT terms. The circle plot incorporates information on $Z$ score, which predicts if a pathway will be upregulated or downregulated. The $Z$ score is calculated by taking the number of upregulated statistically significant genes, subtracting the number of downregulated statistically significant genes, and dividing this number by the square root of the number of genes in each pathway. Additionally, the circle graph highlights the number of upregulated and downregulated genes found in each term, as well as the $P$ value of the GO-term. The chord plot was generated utilizing GOChord and represents the association of 18 significantly differentially expressed genes and the top 8 GO-FAT terms. The chord plot also highlights the $\log _{2}$ fold change associated with each gene.

A volcano plot was generated with ggplot2 (http://ggplot2.org/) and shows the differential gene

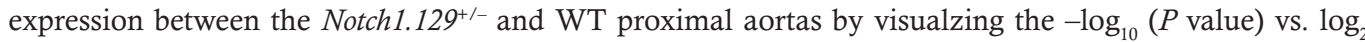
fold change.

Human tissue. AscAA human tissue sections were received from the National Registry of Genetically Triggered Thoracic Aortic Aneurysms and Cardiovascular Conditions (GenTAC) (89). Normal human tissue was procured slightly superior to the STJ in a collaboration with the Davis Heart and Lung Research Institute (The Ohio State University) and Lifeline of Ohio (Columbus, Ohio, USA). Frozen tissue was obtained and fixed in $4 \%$ paraformaldehyde at $4^{\circ} \mathrm{C}$ overnight. Fixed tissue was embedded in paraffin and sectioned at $5 \mu \mathrm{m}$.

Statistics. Statistical analysis was performed in Graphpad Prism 7 software using Student's $t$ tests. Statistical significance was determined based on a $P<0.05$. For RNA-seq, 2-way ANOVA was performed on the $\log _{2}$ RPKM data for the 21,954 detectable mouse genes to examine the effect of genotype (WT and Notch $1^{+/-}$) and location (proximal and distal), as well as their interaction on gene expression. Tukey honestly significant difference tests were performed to determine the effect of genotype within each location and the effect of location within each genotype. No Tukey test was run if the average $\log _{2}$ RPKM value for both groups in the pairwise comparison was less than reliable quantification threshold (RQT). ANOVA and Tukey $P$ values across all genes were adjusted to control the FDR (90). Differentially expressed genes were defined as those having a Tukey $P<0.05$ and an expression fold change $\geq 1.5$. All statistical analysis was performed using $\mathrm{R}$ version 3.2.2 statistical computing software.

Study approval. Animal use was approved and monitored on protocol AR12-00025 by the IACUC at the Research Institute at Nationwide Children's Hospital (Columbus, Ohio, USA).

\section{Author contributions}

SNK contributed to experimental design, data collection, analysis, and manuscript preparation. SL contributed to data collection and bioinformatic analysis. JDF and PR contributed to data collection. KNH assisted with experimental design and performed microCT analysis. AJT performed and analyzed mouse blood pressures. PMLJ contributed to experimental design and data collection. FR provided critical reagents. $\mathrm{BL}$ assisted with experimental design and data interpretation and made critical contributions to the manuscript. VG contributed to experimental design, data analysis, and manuscript preparation.

\section{Acknowledgments}

The authors would like to acknowledge Darian Bauer for her technical assistance in management of the mouse colony for this project, Terri Shaffer for technical assistance with microCT imaging, and Lifeline of Ohio for the assistance with collection of normal human tissue. The GenTAC Registry has been supported by US Federal Government contracts HHSN268200648199C and HHSN268201000048C from the National 
Heart Lung and Blood Institute and the National Institute of Arthritis and Musculoskeletal and Skin Diseases (Bethesda, Maryland, USA). SNK was supported by grant TL1TR001069 from the National Center for Advancing Translational Sciences. The content is solely the responsibility of the authors and does not necessarily represent the official views of the National Center for Advancing Translational Sciences or the NIH. This project was also supported by NIH/National Heart Lung Blood Institute (R01-HL121797), Saving tiny Hearts Society, and Nationwide Children's Hospital Research Institute to Vidu Garg.

Address correspondence to: Vidu Garg, Center for Cardiovascular Research and The Heart Center, Nationwide Children's Hospital, Room WB4221 Columbus, Ohio 43205, USA. Phone: 614.355.5710; Email: vidu.garg@nationwidechildrens.org.

1. Olsson C, Thelin S, Ståhle E, Ekbom A, Granath F. Thoracic aortic aneurysm and dissection: increasing prevalence and improved outcomes reported in a nationwide population-based study of more than 14,000 cases from 1987 to 2002 . Circulation. 2006;114(24):2611-2618.

2. Andelfinger G, Loeys B, Dietz H. A Decade of Discovery in the Genetic Understanding of Thoracic Aortic Disease. Can J Cardiol. 2016;32(1):13-25.

3. Kainulainen K, Pulkkinen L, Savolainen A, Kaitila I, Peltonen L. Location on chromosome 15 of the gene defect causing Marfan syndrome. N Engl J Med. 1990;323(14):935-939.

4. Dietz HC, et al. Marfan syndrome caused by a recurrent de novo missense mutation in the fibrillin gene. Nature. 1991;352(6333):337-339.

5. Lee B, et al. Linkage of Marfan syndrome and a phenotypically related disorder to two different fibrillin genes. Nature. 1991;352(6333):330-334.

6. Maslen CL, Corson GM, Maddox BK, Glanville RW, Sakai LY. Partial sequence of a candidate gene for the Marfan syndrome. Nature. 1991;352(6333):334-337.

7. Loeys BL, et al. A syndrome of altered cardiovascular, craniofacial, neurocognitive and skeletal development caused by mutations in TGFBR1 or TGFBR2. Nat Genet. 2005;37(3):275-281.

8. Bertoli-Avella AM, et al. Mutations in a TGF- $\beta$ ligand, TGFB3, cause syndromic aortic aneurysms and dissections. $J$ Am Coll Cardiol. 2015;65(13):1324-1336.

9. Lindsay ME, et al. Loss-of-function mutations in TGFB2 cause a syndromic presentation of thoracic aortic aneurysm. Nat Genet. 2012;44(8):922-927.

10. van de Laar IM, et al. Mutations in SMAD3 cause a syndromic form of aortic aneurysms and dissections with early-onset osteoarthritis. Nat Genet. 2011;43(2):121-126.

11. Barbey F, et al. Aortic remodelling in Fabry disease. Eur Heart J. 2010;31(3):347-353.

12. Lopez L, et al. Turner syndrome is an independent risk factor for aortic dilation in the young. Pediatrics. 2008;121(6):e1622-e1627.

13. Pepin M, Schwarze U, Superti-Furga A, Byers PH. Clinical and genetic features of Ehlers-Danlos syndrome type IV, the vascular type. N Engl J Med. 2000;342(10):673-680.

14. Guo DC, et al. Mutations in smooth muscle alpha-actin (ACTA2) lead to thoracic aortic aneurysms and dissections. Nat Genet. 2007;39(12):1488-1493.

15. Zhu L, et al. Mutations in myosin heavy chain 11 cause a syndrome associating thoracic aortic aneurysm/aortic dissection and patent ductus arteriosus. Nat Genet. 2006;38(3):343-349.

16. Guo DC, et al. LOX Mutations Predispose to Thoracic Aortic Aneurysms and Dissections. Circ Res. 2016;118(6):928-934.

17. Lee VS, et al. Loss of function mutation in LOX causes thoracic aortic aneurysm and dissection in humans. Proc Natl Acad Sci USA. 2016;113(31):8759-8764.

18. Kuang SQ, et al. FOXE3 mutations predispose to thoracic aortic aneurysms and dissections. J Clin Invest. 2016;126(3):948-961.

19. Girdauskas E, Borger MA, Secknus MA, Girdauskas G, Kuntze T. Is aortopathy in bicuspid aortic valve disease a congenital defect or a result of abnormal hemodynamics? A critical reappraisal of a one-sided argument. Eur J Cardiothorac Surg. 2011;39(6):809-814.

20. Bäck M, Gasser TC, Michel JB, Caligiuri G. Biomechanical factors in the biology of aortic wall and aortic valve diseases. Cardiovasc Res. 2013;99(2):232-241.

21. Yasuda $\mathrm{H}$, et al. Failure to prevent progressive dilation of ascending aorta by aortic valve replacement in patients with bicuspid aortic valve: comparison with tricuspid aortic valve. Circulation. 2003;108 Supp1 1:II291-II294.

22. Mjaatvedt CH, et al. The outflow tract of the heart is recruited from a novel heart-forming field. Dev Biol. 2001;238(1):97-109.

23. Waldo KL, et al. Conotruncal myocardium arises from a secondary heart field. Development. 2001;128(16):3179-3188

24. Kelly RG, Brown NA, Buckingham ME. The arterial pole of the mouse heart forms from Fgf10-expressing cells in pharyngeal mesoderm. Dev Cell. 2001;1(3):435-440.

25. Kirby ML, Gale TF, Stewart DE. Neural crest cells contribute to normal aorticopulmonary septation. Science. 1983;220(4601):1059-1061.

26. Jiang X, Rowitch DH, Soriano P, McMahon AP, Sucov HM. Fate of the mammalian cardiac neural crest. Development. 2000;127(8):1607-1616

27. Garg V, et al. Mutations in NOTCH1 cause aortic valve disease. Nature. 2005;437(7056):270-274.

28. McKellar SH, Tester DJ, Yagubyan M, Majumdar R, Ackerman MJ, Sundt TM. Novel NOTCH1 mutations in patients with bicuspid aortic valve disease and thoracic aortic aneurysms. J Thorac Cardiovasc Surg. 2007;134(2):290-296.

29. Mohamed SA, et al. Novel missense mutations (p.T596M and p.P1797H) in NOTCH1 in patients with bicuspid aortic valve. Biochem Biophys Res Commun. 2006;345(4):1460-1465. 
30. Proost D, et al. Performant Mutation Identification Using Targeted Next-Generation Sequencing of 14 Thoracic Aortic Aneurysm Genes. Hum Mutat. 2015;36(8):808-814.

31. Swiatek PJ, Lindsell CE, del Amo FF, Weinmaster G, Gridley T. Notch1 is essential for postimplantation development in mice. Genes Dev. 1994;8(6):707-719.

32. Krebs LT, et al. Notch signaling is essential for vascular morphogenesis in mice. Genes Dev. 2000;14(11):1343-1352.

33. Jain R, et al. Cardiac neural crest orchestrates remodeling and functional maturation of mouse semilunar valves. J Clin Invest. 2011;121(1):422-430

34. Bosse K, et al. Endothelial nitric oxide signaling regulates Notch1 in aortic valve disease. J Mol Cell Cardiol. 2013;60:27-35.

35. High FA, et al. Murine Jagged1/Notch signaling in the second heart field orchestrates Fgf8 expression and tissue-tissue interactions during outflow tract development. J Clin Invest. 2009;119(7):1986-1996.

36. Koenig SN, Bosse KM, Nadorlik HA, Lilly B, Garg V. Evidence of Aortopathy in Mice with Haploinsufficiency of Notch1 in Nos3-Null Background. J Cardiovasc Dev Dis. 2015;2(1):17-30.

37. Koenig SN, Bosse K, Majumdar U, Bonachea EM, Radtke F, Garg V. Endothelial Notch1 is required for proper development of the semilunar valves and cardiac outflow tract. J Am Heart Assoc. 2016;5(4):e003075.

38. Wang Y, et al. Notch-Tnf signalling is required for development and homeostasis of arterial valves. Eur Heart J. 2017;38(9):675-686.

39. Judge DP, et al. Evidence for a critical contribution of haploinsufficiency in the complex pathogenesis of Marfan syndrome. J Clin Invest. 2004;114(2):172-181.

40. Sciacca S, Pilato M, Mazzoccoli G, Pazienza V, Vinciguerra M. Anti-correlation between longevity gene SirT1 and Notch signaling in ascending aorta biopsies from patients with bicuspid aortic valve disease. Heart Vessels. 2013;28(2):268-275.

41. Grego-Bessa J, et al. Notch signaling is essential for ventricular chamber development. Dev Cell. 2007;12(3):415-429.

42. Timmerman LA, et al. Notch promotes epithelial-mesenchymal transition during cardiac development and oncogenic transformation. Genes Dev. 2004;18(1):99-115.

43. Theodoris CV, et al. Human disease modeling reveals integrated transcriptional and epigenetic mechanisms of NOTCH1 haploinsufficiency. Cell. 2015;160(6):1072-1086.

44. White MP, et al. NOTCH1 regulates matrix gla protein and calcification gene networks in human valve endothelium. $J$ Mol Cell Cardiol. 2015;84:13-23.

45. Kisanuki YY, Hammer RE, Miyazaki J, Williams SC, Richardson JA, Yanagisawa M. Tie2-Cre transgenic mice: a new model for endothelial cell-lineage analysis in vivo. Dev Biol. 2001;230(2):230-242.

46. Chen Q, et al. miRNA-34a reduces neointima formation through inhibiting smooth muscle cell proliferation and migration. J Mol Cell Cardiol. 2015;89(Pt A):75-86.

47. Li Y, et al. Smooth muscle Notch1 mediates neointimal formation after vascular injury. Circulation. 2009;119(20):2686-2692.

48. Liang M, et al. Migration of smooth muscle cells from the arterial anastomosis of arteriovenous fistulas requires Notch activation to form neointima. Kidney Int. 2015;88(3):490-502.

49. Xin HB, Deng KY, Rishniw M, Ji G, Kotlikoff MI. Smooth muscle expression of Cre recombinase and eGFP in transgenic mice. Physiol Genomics. 2002;10(3):211-215.

50. Verzi MP, McCulley DJ, De Val S, Dodou E, Black BL. The right ventricle, outflow tract, and ventricular septum comprise a restricted expression domain within the secondary/anterior heart field. Dev Biol. 2005;287(1):134-145.

51. Danielian PS, Muccino D, Rowitch DH, Michael SK, McMahon AP. Modification of gene activity in mouse embryos in utero by a tamoxifen-inducible form of Cre recombinase. Curr Biol. 1998;8(24):1323-1326.

52. Long X, Creemers EE, Wang DZ, Olson EN, Miano JM. Myocardin is a bifunctional switch for smooth versus skeletal muscle differentiation. Proc Natl Acad Sci USA. 2007;104(42):16570-16575.

53. Sawada H, Rateri DL, Moorleghen JJ, Majesky MW, Daugherty A. Smooth Muscle Cells Derived From Second Heart Field and Cardiac Neural Crest Reside in Spatially Distinct Domains in the Media of the Ascending Aorta-Brief Report. Arterioscler Thromb Vasc Biol. 2017;37(9):1722-1726.

54. Kim JH, et al. Integration of gene-expression profiles and pathway analysis in ascending thoracic aortic aneurysms. Ann Vasc Surg. 2010;24(4):538-549.

55. Patuzzo C, et al. A Preliminary microRNA Analysis of Non Syndromic Thoracic Aortic Aneurysms. Balkan J Med Genet. 2012;15(Suppl):51-55.

56. Sinha S, Iyer D, Granata A. Embryonic origins of human vascular smooth muscle cells: implications for in vitro modeling and clinical application. Cell Mol Life Sci. 2014;71(12):2271-2288.

57. Waldo KL, et al. Secondary heart field contributes myocardium and smooth muscle to the arterial pole of the developing heart. Dev Biol. 2005;281(1):78-90

58. Bargehr J, et al. Embryological Origin of Human Smooth Muscle Cells Influences Their Ability to Support Endothelial Network Formation. Stem Cells Transl Med. 2016;5(7):946-959.

59. Haimovici H, Maier N. Fate Of Aortic Homografts In Canine Atherosclerosis. 3. Study Of Fresh Abdominal And Thoracic Aortic Implants Into Thoracic Aorta: Role Of Tissue Susceptibility In Atherogenesis. Arch Surg. 1964;89:961-969.

60. Rosenquist TH, Kirby ML, van Mierop LH. Solitary aortic arch artery. A result of surgical ablation of cardiac neural crest and nodose placode in the avian embryo. Circulation. 1989;80(5):1469-1475

61. Topouzis S, Majesky MW. Smooth muscle lineage diversity in the chick embryo. Two types of aortic smooth muscle cell differ in growth and receptor-mediated transcriptional responses to transforming growth factor-beta. Dev Biol. 1996;178(2):430-445

62. Muzumdar MD, Tasic B, Miyamichi K, Li L, Luo L. A global double-fluorescent Cre reporter mouse. Genesis. 2007;45(9):593-605

63. Devine WP, Wythe JD, George M, Koshiba-Takeuchi K, Bruneau BG. Early patterning and specification of cardiac progenitors in gastrulating mesoderm. Elife. 2014;3:e03848

64. Liu Z, et al. Second-generation Notch1 activity-trap mouse line (N1IP::CreHI) provides a more comprehensive map of cells experiencing Notch1 activity. Development. 2015;142(6):1193-1202.

65. Golledge J, Clancy P, Maguire J, Lincz L, Koblar S. The role of tenascin C in cardiovascular disease. Cardiovasc Res. 2011;92(1):19-28.

66. Hedin U, Holm J, Hansson GK. Induction of tenascin in rat arterial injury. Relationship to altered smooth muscle cell pheno- 
type. Am J Pathol. 1991;139(3):649-656.

67. Jones PL, Rabinovitch M. Tenascin-C is induced with progressive pulmonary vascular disease in rats and is functionally related to increased smooth muscle cell proliferation. Circ Res. 1996;79(6):1131-1142.

68. Della Corte A, et al. Spatiotemporal patterns of smooth muscle cell changes in ascending aortic dilatation with bicuspid and tricuspid aortic valve stenosis: focus on cell-matrix signaling. J Thorac Cardiovasc Surg. 2008;135(1):18.e1.

69. Kimura T, et al. Tenascin C protects aorta from acute dissection in mice. Sci Rep. 2014;4:4051.

70. Matsumoto K, Satoh K, Maniwa T, Tanaka T, Okunishi H, Oda T. Proteomic comparison between abdominal and thoracic aortic aneurysms. Int J Mol Med. 2014;33(4):1035-1047.

71. Trescher K, et al. Type A dissection and chronic dilatation: tenascin-C as a key factor in destabilization of the aortic wall. Interact Cardiovasc Thorac Surg. 2013;17(2):365-370.

72. Remus EW, et al. The role of lysyl oxidase family members in the stabilization of abdominal aortic aneurysms. Am J Physiol Heart Circ Physiol. 2012;303(8):H1067-H1075.

73. Busnadiego O, et al. Elevated expression levels of lysyl oxidases protect against aortic aneurysm progression in Marfan syndrome. J Mol Cell Cardiol. 2015;85:48-57.

74. Zou S, Ren P, Nguyen M, Coselli JS, Shen YH, LeMaire SA. Notch signaling in descending thoracic aortic aneurysm and dissection. PLoS ONE. 2012;7(12):e52833.

75. Wang YW, Ren HL, Wang HF, Li FD, Li HH, Zheng YH. Combining detection of Notch1 and tumor necrosis factor- $\alpha$ converting enzyme is a reliable biomarker for the diagnosis of abdominal aortic aneurysms. Life Sci. 2015;127:39-45.

76. Padang R, Bagnall RD, Richmond DR, Bannon PG, Semsarian C. Rare non-synonymous variations in the transcriptional activation domains of GATA5 in bicuspid aortic valve disease. J Mol Cell Cardiol. 2012;53(2):277-281.

77. Bonachea EM, et al. Rare GATA5 sequence variants identified in individuals with bicuspid aortic valve. Pediatr Res. 2014;76(2):211-216

78. Laforest B, Andelfinger G, Nemer M. Loss of Gata5 in mice leads to bicuspid aortic valve. J Clin Invest. 2011;121(7):2876-2887.

79. Michelena HI, et al. Natural history of asymptomatic patients with normally functioning or minimally dysfunctional bicuspid aortic valve in the community. Circulation. 2008;117(21):2776-2784.

80. Tzemos N, et al. Outcomes in adults with bicuspid aortic valves. JAMA. 2008;300(11):1317-1325

81. Radtke F, et al. Deficient T cell fate specification in mice with an induced inactivation of Notch1. Immunity. 1999;10(5):547-558.

82. Soriano P. Generalized lacZ expression with the ROSA26 Cre reporter strain. Nat Genet. 1999;21(1):70-71.

83. Trapnell C, Pachter L, Salzberg SL. TopHat: discovering splice junctions with RNA-Seq. Bioinformatics. 2009;25(9):1105-1111.

84. Langmead B, Trapnell C, Pop M, Salzberg SL. Ultrafast and memory-efficient alignment of short DNA sequences to the human genome. Genome Biol. 2009;10(3):R25.

85. Delhomme N, Padioleau I, Furlong EE, Steinmetz LM. easyRNA-seq: a Bioconductor package for processing RNA-Seq data. Bioinformatics. 2012;28(19):2532-2533.

86. de Hoon MJ, Imoto S, Nolan J, Miyano S. Open source clustering software. Bioinformatics. 2004;20(9):1453-1454

87. Huang da W, Sherman BT, Lempicki RA. Systematic and integrative analysis of large gene lists using DAVID bioinformatics resources. Nat Protoc. 2009;4(1):44-57.

88. Walter W, Sánchez-Cabo F, Ricote M. GOplot: an R package for visually combining expression data with functional analysis Bioinformatics. 2015;31(17):2912-2914.

89. Eagle KA, GenTAC Consortium. Rationale and design of the National Registry of Genetically Triggered Thoracic Aortic Aneurysms and Cardiovascular Conditions (GenTAC). Am Heart J. 2009;157(2):319-326.

90. Benjamini Y, Hochberg Y. Controlling the false discovery rate: a practical and powerful approach to multiple testing. $J R$ Stat Soc Series B Stat Methodol. 1995;57(1):289-300. 